\title{
EIT Crinkles as Evidence for the Breakout Model of Solar Eruptions
}

Alphonse C. Sterling ${ }^{1}$ and Ronald L. Moore

NASA/Marshall Space Flight Center, SD50/Space Science Department, Huntsville, AL 35812 asterling@solar.stanford.edu

ron.moore@msfc.nasa.gov

${ }^{1} \mathrm{NRC}$ - MSFC Research Associate 
Received —_ accepted

Submitted to The Astrophysical Journal (April 20, 2001 Version) 


\begin{abstract}
We present observations of two homologous flares in NOAA active region 8210 occurring on 1998 May 1 and May 2, using EUV data from the EUV Imaging Telescope (EIT) on the Solar and Heliospheric Observatory ( $S O H O)$, high-resolution and high-time cadence images from the soft X-ray telescope (SXT) on Yohkoh, images or fluxes from the hard X-ray telescope (HXT) on Yohkoh and the BATSE experiment on the Compton Gamma Ray Observatory (CGRO), and Ca XIX soft X-ray spectra from the Bragg crystal spectrometer (BCS) on Yohkoh. Magnetograms indicate that the flares occurred in a complex magnetic topology, consisting of an emerging flux region (EFR) sandwiched between a sunspot to the west and a coronal hole to the east. In an earlier study we found that in EIT images, both flaring episodes showed the formation of a crinkle-like pattern of emission ("EIT crinkles") occurring in the coronal hole vicinity, well away from a central "core field" area near the EFR-sunspot boundary. With our expanded data set, here we find that most of the energetic activity occurs in the core region in both events, with some portions of the core brightening shortly after the onset of the EIT crinkles, and other regions of the core brightening several minutes later, coincident with a burst of hard X-rays: there are no obvious core brightenings prior to the onset of the EIT crinkles. These timings are consistent with the "breakout model" of solar eruptions, whereby the emerging flux is initially constrained by a system of overlying magnetic field lines, and is able to erupt only after an opening develops in the overlying fields as a consequence of magnetic reconnection at a magnetic null point. In our case, the EIT crinkles would be a signature of this pre-impulsive-phase magnetic reconnection, and brightening of the core only occurs after the core fields begin to escape through the newly-created opening in the overlying fields. Morphology in soft X-ray images and properties in hard X-rays differ between the two events, with complexities that preclude a simple determination of the dynamics in the core at the times of eruption. From the BCS spectra, we find that the core region expends energy at a rate of $\sim 10^{26} \mathrm{erg} \mathrm{s}^{-1}$ during the time of the growth of the EIT crinkles; this rate is an upper limit to energy expended in the reconnections opening the overlying fields. Energy losses occur at an order-of-magnitude higher rate near the time of the peak of the events. There is little evidence of asymmetry in the spectra, consistent with the majority of the mass flows occurring normal to the line-of-sight. Both events have similar electron temperature dependencies on time.
\end{abstract}

Subject headings: Sun: corona — Sun: flares — Sun: particle emission — Sun: X-rays, gamma rays — Sun: UV radiation 


\section{Introduction}

Eruptive phenomena on the Sun, such as flares and coronal mass ejections (CMEs) originate from the conversion of magnetic energy into thermal and mechanical energy. Just how, and with what rate, this conversion takes place, however, is still a mystery; resolving this mystery is key to understanding the eruption mechanism and, eventually, accurately forecasting when eruptions will occur. Analysis of data obtained from space-based satellites, including Skylab, P78-1, and SMM, in the 1970's and 1980's resulted in an epochal advance in our understanding of these phenomena. Work on data from currently-flying missions has further expanded and enhanced our knowledge of flare and CME morphology and physics. A "standard model" developed from these observations holds that much of the energy in these events is released in a core magnetic field region which is enveloped in a canopy of overlying magnetic fields. Some process, such as reconnection or an instability, leads to the eruption of the core fields. These erupting core fields displace and stretch out the overlying magnetic field lines, and as the eruption proceeds, the stretched field lines re-close via reconnection beneath the erupting fields. The reconnected fields form magnetic loops which emit strongly in soft X-rays and EUV, while the locations where these loops meet the photosphere (the footpoints) are sources for the H-alpha ribbons and much of the flare-associated hard X-ray emission.

In this paper we are concerned mainly with what happens prior to and during the time of the primary energy release; in particular, we examine data germane to deciphering where the source of the initial disruption of the core field lies. One possible such source is inside the core field itself. The "tether cutting" model (e.g., Moore \& LaBonte 1980; Sturrock 1989; Moore et al. 1997; 2001) is based on this assumption, taking the approach that magnetic reconnection between flux elements within the core field results in the eruption. A second possible source is in the surrounding fields, as proposed in the "breakout model" (Antiochos 1998; Antiochos, DeVore, \& Klimchuk 1999), which maintains that the eruption ensues only after a breach occurs due to magnetic reconnection at a magnetic null point in a broad system of overlying restraining fields. Tether-cutting is fundamentally a bipolar mechanism, whereas the breakout model holds that a multipolar magnetic topology is essential to the eruption.

Recently, Sterling \& Moore (2001) studied a series of homologous solar flares in an active region designated NOAA 8210, primarily using data from the EUV Imaging Telescope (EIT) and the Michelson Doppler Imager (MDI) on the Solar and Heliospheric Observatory (SOHO), and low time- and spatial-resolution soft X-ray images from the Soft X-ray Telescope (SXT) on the Yohkoh satellite. Early in each flaring episode, EIT $195 \AA$ images show the formation of transient, small-scale unresolved patches of emission well away from a central core flaring region, which Sterling \& Moore called EIT crinkles. These EIT crinkles first propagate away from (with velocity $\sim 20 \mathrm{~km} \mathrm{~s}^{-1}$ ), and then fade in toward the central core region. Concurrent with the EIT crinkle formation, the low-resolution SXT images show faint, transient brightening of features connecting the central core region with the locations of the EIT crinkles, indicating that the crinkles are the lower-temperature footpoints of newly heated coronal loops. At later times, post-flare loops develop in soft X-rays and EUV, initially low in the core region and then growing in extent from that core. Sterling \& Moore inferred that they were observing dynamics associated with a core field enveloped in overlying fields, with the EIT crinkles resulting from reconnections between core fields and the overlying fields. With their data alone, however, they were not able to examine in detail the timings of the dynamics associated with the core region itself, and hence they did not make a judgment as to whether this sequence of events favored either the tether-cutting model or the breakout 
model. In this paper, we address these questions more closely for two of the homologous events, by combining high-time cadence and high-resolution SXT images, hard X-ray data from the Hard X-ray Telescope (HXT) on Yohkoh and the Burst and Transient Source Experiment (BATSE) on the Compton Gamma Ray Observatory (CGRO), and data from the Ca XIX channel of the Bragg Crystal Spectrometer (BCS) experiment on Yohkoh, in addition to the EIT and MDI SOHO data.

So far, no systematic search has been carried out for EIT crinkle events, so we do not know how common they are. They do appear to require specific magnetic field properties, e.g., particularly strong enveloping magnetic fields. Nonetheless, the homologous events of AR 8210 provide an important laboratory for studying eruptive events, since signatures of the enveloping fields are clearly identifiable. If this type of magnetic setup is key to the eruptive process, as envisioned by, for example, the breakout model, then aspects of the AR 8210 events could be common to many flares, albeit at varying levels of prominence.

\section{Instrumentation and Data}

Our previous analysis of AR 8210 (Sterling \& Moore 2001) was based mainly on a sequence of EIT $195 \AA$ images; here we will use these same images to set the context for our further studies. EIT actually utilizes four filters, each producing full-Sun images with $2^{\prime \prime} .6$ pixels (Delaboudiniere

et al. 1995). We use the $195 \AA$ Fe XII filter since it has the most appropriate time resolution (about 17 minutes) for our purposes. Yohkoh's SXT (Tsuneta et al. 1991) uses analysis filters spanning the approximate wavelength range 3-45 $\AA$, effectively detecting coronal plasmas at temperatures $\gtrsim 2-3$ MK. SXT takes either full-frame (full disk) images with a pixel resolution of $9^{\prime \prime} .8-$ or $4^{\prime \prime} .9$, or higher resolution (2".5 pixels) "partial frame" images which have a limited field of view. Our SXT studies in Sterling \& Moore (2001) were based on full-frame images, which generally have a time cadence $\sim 10 \mathrm{~min}$. In the current work we will primarily examine Al.1-filter partial-frame images with time cadence $\sim 10 \mathrm{~s}$. Although the Al.1 (aluminum) filter is a "thin" filter (i.e., extends to softer X-rays than thicker filters), we found images in the "thick" (i.e., extending to shorter wavelengths) beryllium filter to be qualitatively similar to those taken with the aluminum filter.

We use HXT and BATSE data for hard X-ray information. HXT observes with $\sim 0.5 \mathrm{~s}$ resolution in four energy bands: $13.9-22.7 \mathrm{keV}, 22.7-32.7 \mathrm{keV}, 32.7-52.7 \mathrm{keV}$, and $52.7-$ $92.8 \mathrm{keV}$, referred to as the L-, M1-, M2-, and H-bands, respectively. Our BATSE data are from its Discriminator Large Area (DISCLA) detectors, which has four energy channels and $1.024 \mathrm{~s}$ resolution; here we use data from the $25-50 \mathrm{keV}, 50-100 \mathrm{keV}$ channels. BATSE's signal-to-noise level is better than that of HXT, but we present data from both instruments since data coverage is sometimes better with HXT, and because we present images synthesized from the HXT data. Kosugi et al. (1991) discuss details of the HXT instrument, and Fishman et al. (1989) describe the BATSE instrument.

Yohkoh's BCS consists of four spectral channels; here we restrict our analysis to the He-like Ca XIX channel, which covers the resonance line and associated satellite lines of He-like calcium (roughly 3.16-3.19 $\AA$ ). Culhane et al. (1991) give details of the instrument, and we provide information specific to our current studies in $\S 4.2$.

AR 8210 appeared on the Sun in 1998 April, and was near disk center on 1998 May 1-2, when a series of flares with the same general appearance in EIT $195 \AA$ images occurred. A review of the EIT data show that there were at least three episodes in this "morphologically homologous" 
sequence, with peak flux in GOES soft X-ray channels occurring near 17:57 UT and 22:54 UT on May 1, and 05:00 UT on May 2; these events were discussed in detail in Sterling \& Moore (2001). A preliminary analysis (O. C. St. Cyr 2000, private communication) of data from the Large Angle and Spectrometric Coronagraph Experiment (LASCO) experiment on SOHO shows halo CMEs (indicating that the CMEs were directed along the Sun-Earth line-of-site, e.g., Howard et al. 1982; Hudson et al. 1998; Webb et al. 2000) that were likely to be associated with the second and third events of this sequence. Of these, the halo CME likely to have been associated with the second event was first detected while in progress at 23:40 UT on May 1, following a data gap in the LASCO data extending from 16:27 - 23:40 UT. The halo CME likely to have been associated with the third event was first seen at 05:31 UT on May 2. Due to the data gap, we are unable to confirm that there was a CME associated with the first event. All three of these events, however, showed the same general appearance in EIT, including the development of systems of post-flare loops, which are believed to be signatures of CME expulsion (e.g., Hirayama 1974; Kahler 1977). Therefore, we expect that

each of these events was ejective. Weaker episodes in the same sequence may have occurred in EIT images near 06:30 UT and 12:30 UT on May 1.

We will present in detail the events at 22:54 UT on May 1, and at 05:00 UT on May 2 (hereafter referred to as the May 1 and May 2 events, respectively). We do not give a detailed account of the 17:57 UT May 1 event since it begins during Yohkoh night, and data coverage in hard X-rays and BCS are not satisfactory for that event; we will, however, discuss it briefly in $\S 6$ in comparison with the two better-observed events. We also miss the start of the intensity rise in SXT for the May 2 event due to Yohkoh night, but coverage in other instruments is adequate. Figure 1 presents GOES data covering the May 1 and May 2 events, with the hashed regions indicating times when Yohkoh was in spacecraft night. Based on the position of the resonance line in the Ca XIX spectra (whose apparent wavelength varies with the latitude of the emitting source) and a full-frame SXT image, we found that the GOES C1 event peaking near 04:35 UT on May 2 is associated with an active region further north than AR 8210, and thus is not a factor in our analysis.

\section{EIT Crinkles: Observations and a Schematic Picture}

We first summarize observations of EIT crinkles for the two events of Figure 1, and present a schematic interpretation of the observations, viz., the picture suggested by Sterling \& Moore (2001). Figure 2 shows selected SXT (Figs $2 \mathrm{a}$ and $2 \mathrm{~b}$ ) and EIT (Figs 2c-2f) images from the May 1 event, overlaid with an MDI magnetogram in four panels. Panels on the left are from prior to the onset of the eruption, and panels on the right are from near times of the earliest indication of the eruption. First we consider the magnetic field geometry of the region: A negative-flux sunspot is visible in the northwest (upper right in the figure) of the magnetogram, with positive polarity regions nearly surrounding the spot. Inspection of magnetograms from previous days indicates that the positive flux butted up on the southeast side of the spot is part of a newly-emerging, emerging flux region (EFR), where the negative flux is very close to, or inside of, the spot. Further to the east is a region of weak negative flux which outlines a coronal hole-like area, which is best visible in the EIT images (Figs 2e and 2f). Thus, the magnetic geometry of the region consists of an EFR sandwiched between the strong fields of a sunspot to the northwest and a weak, open, coronal hole-like field to the east.

Next we consider soft X-rays. Prior to event onset, the SXT image (Fig. 2a) shows a bright, S-shaped feature close to the core-field region associated with the magnetic neutral line; we will 
see in $\S 4$ that the most energetic aspects of the flare occur in this core-field region. Such S-shaped (or inverse S-shaped) features are known as "sigmoids," and have been suggested as soft X-ray coronal precursors to eruptive events and CMEs (e.g., Canfield, Hudson, \& McKenzie 1999; Glover et al. 2000; Sterling 2000). Sterling et al. (2000) found that the central portion of sigmoids tend to follow magnetic inversion lines. In Figure 2a, the sigmoid runs roughly parallel to, but slightly to the east of the inversion line. Apparently, the core field threading the sigmoid and straddling the neutral line is forced to lean to the east by the neighboring field of the sunspot. Surrounding the sigmoid in Figure 2a is a broader coronal structure, similar to the "anemone regions" seen in SXT data (Shibata et al. 1994). Near the time of event onset (Fig. 2b), the sigmoid shape is no longer obvious, and there are soft X-ray brightenings extending from the core region out to the region of the coronal hole-like region in the east. The previous anemone fields are not obvious in this image.

Figure 2c is an EIT $195 \AA$ image taken nearly concurrent with the Figure 2a SXT image. Sigmoids are typically less prominent in EIT 195 than in SXT (Sterling 2000), as is the case here. In Figures 2d and 2f, new brightenings are forming at locations corresponding to the ends of some of the SXT extensions (arrows in Fig. 2f); these features are the EIT crinkles. They appear to be footpoints of the hotter SXT loops, with at least one prominent crinkle forming in a clump of negative magnetic polarity (southernmost arrow in Fig. 2f).

In Figure 3 we show a more complete EIT sequence for the event of Figure 2. Figure 3a shows the core region in the west, an anemone region in the middle, and the coronal hole in the east. Figure $3 \mathrm{~b}$ shows the first hint of the EIT crinkles developing in the southwest (cf. lower arrow in Fig. 2f); these crinkles are more obvious by 22:05 UT (Fig. 3c). Crinkles in the northeast (near the upper arrow in Fig. 1f) may start near this time also, but they are most obvious in Figures 3d and 3e. After developing and extending westward, the crinkles fade at later times, becoming parts of more-diffuse structures. This is consistent with the EIT crinkles initially being the cooler footpoints of hotter soft X-ray loops, and these hotter loops eventually cooling to EUV temperatures (Sterling \& Moore 2001). Meanwhile, EIT brightenings in the core region become prominent in Figure 3d; these brightenings develop into a system of postflare loops in Figures 3e and $3 \mathrm{f}$.

In Figure 4 we show a similar sequence for the event beginning near 4:30 UT on 1998 May 2. EIT crinkles develop in locations similar to those in Figure 3, and are most prominent in Figures $4 \mathrm{~b}-4 \mathrm{~d}$. In the core region brightenings are obvious in Figure $4 \mathrm{c}$, and postflare loops from this region are visible in Figure $4 \mathrm{f}$.

Figure 5 shows Sterling and Moore's (2001) schematic interpretation of these observations, with the magnetic setup motivated by the magnetogram of Figure 2: a sunspot on the right, coronal hole on the left, and an EFR in the middle. Sterling and Moore (2001) suggested that each episode begins when the upper portion of the sigmoid erupts outward. This erupting core magnetic field reconnects with the surrounding, overlying magnetic fields ("external reconnection"). Energization of the newly-reconnected fields results in the transient soft X-ray brightenings and excites enhanced EUV emission at the footpoints of these reconnected fields, resulting in the EIT crinkles; that is, the EIT crinkles are the feet of new SXT loops. When the erupting core field moves outward, the EIT crinkles move outward. Because of the magnetic field geometry of this particular region, subsequent reconnections can occur after the core field escapes as part of a CME; these subsequent reconnections occur beginning with the farthest-reaching anemone fields and progress inward toward the core field, resulting in the inward retraction of the outer lobe of the anemone as seen in EIT (as the crinkles fade), and a reinstatement of the original geometry, accounting for the homologous nature of the eruptions. Meanwhile, reconnection also occurs within the closed EFR magnetic fields 
("internal reconnection") resulting in the bright flare loops seen in Figures 3e, 3f, 4e and 4f; thus the fields associated with the internal reconnection closely follow the scenario of the standard model discussed in $\S 1$.

We will reconsider this schematic in $\S 6$ after a closer look at the evolution of the core region in the two events of Figure 1. We will update our description of the initiation of the eruption in light of the new observations, but the remainder of the story will largely remain intact.

EIT crinkles have similarities with a newly-named phenomena, "moss," observed with the TRACE satellite (Berger et al. 1999; Fletcher \& De Pontieu 1999). Both are near-1 MK features which appear to be footpoints of hotter loops seen with SXT. Moss, however, is associated with non-flaring active region loops, and is likely to be a consequence of a "steady state" coronal heating source (Martens, Kankelborg, \& Berger 2000). EIT crinkles are associated with flares, and show evidence of propagation with time. They are also much more transient and are associated with shorter-lived SXT loops than the moss so far discussed. EIT crinkles appear to be similar to remote flare brightenings (e.g., Tang \& Moore 1982). Our use of the expression "EIT crinkles" is purely descriptive; future observations with TRACE may show them to otherwise be qualitatively identical to moss, in which case EIT crinkles could be identified as "dynamic moss" or "flare-associated moss." On the other hand, if the EIT crinkles are found to have substantially different properties from the moss, then a more appropriate name may be "EUV crinkles."

\section{Event of 22 UT, 1998 May 1}

\subsection{Soft and Hard X-Ray Data}

We now present a more detailed analysis of the 22 UT, 1998 May 1 EIT crinkle event. Figure 6 shows full-resolution SXT images of the region at various stages of its evolution. Figure 6a is near the time of the lower-resolution image of Figure 2a, and shows that the sigmoid consists of several illuminated loops, although it is still difficult to discern the precise geometry. This image is from before the eruption, with the initial anemone region visible. At the time of Figure $6 \mathrm{~b}$, the soft X-ray extensions (between the core and the EIT crinkles) of Figure $2 \mathrm{~b}$ are faintly visible, and are much clearer in Figure 6c. Meanwhile, the core region shows brightenings beginning from about Figure 6c. Its development continues in the smaller field-of-view images of Figures $6 \mathrm{e}-6 \mathrm{~g}$. Several major loops are visible in these latter images, with complexities that are difficult to categorize. Figures $6 \mathrm{j}$ and $6 \mathrm{k}$ show the development of postflare loops late in the decay phase (cf. Fig. 1). By the time of Figure 61, the postflare loops have faded, and the sigmoid is visible once again. It is likely that many of the components of the sigmoid were present throughout the event, but were obscured by the brightest flaring loops when the intensity was high; Sterling and Moore (2001) reached this conclusion since they found that the sigmoid remained visible even near the peak of the relatively low-intensity eruption near 17:57 UT on 1998 May 1, i.e., the first of the primary eruptions in the morphologically-homologous sequence.

Figure $6 \mathrm{~h}$ shows the same SXT image as in Figures $6 \mathrm{f}$ and $6 \mathrm{~g}$, overlaid with an HXT L-band image from nearly the same time. This image was synthesized using the Maximum Entropy Method (MEM; see Kosugi et al. 1991), and shows two principal source regions. Figure $6 \mathrm{i}$ again shows the same SXT image, this time with boxes denoting principal areas of the region: Box D corresponds to the location of the earliest brightenings in the region visible in Figure 6c, Boxes A and B correspond 
to the locations of the hard X-ray sources of Figure $6 \mathrm{~h}$, and Box $\mathrm{C}$ corresponds to the entire flaring region.

Figure 7a shows lightcurves obtained by summing the intensity in the SXT images corresponding to the boxes in Figure 6i, while Figure 7b shows hard X-ray lightcurves from the HXT L- and M1-bands, plotted on the same timescale as Figure 7a. There are two patterns present in the SXT lightcurves of Figure 7a: Lightcurves $\mathrm{A}$ and $\mathrm{B}$ show a sharp rise in intensity coincident with the onset of the hard X-rays in Figure 7b. Lightcurve D shows a different pattern, with an intensity rise beginning well before the onset of the hard X-rays, and little response to the hard X-rays. Moreover, Figure $7 \mathrm{~b}$ indicates that there are no hard X-rays in the L-band above noise level prior to about 22:34 UT (M1-band data are not available prior to about 22:40 UT, when Yohkoh "flare mode" observations ensued). Thus, the intensity increase of lightcurve D occurs independent of a detectable HXT burst. (No such burst is apparent even if we plot the logarithm of the hard X-ray flux; we plot Fig. 7b on a linear scale in order to compare the hardness of the hard X-rays with those in Fig. 10 below.) Figure 7c shows the more-sensitive BATSE data, indicating no hard X-ray bursts at its noise level between about 22:24 UT (shortly after the CGRO spacecraft emerged from night) and 22:34 UT.

\subsection{BCS Spectra}

We fit the BCS Ca XIX spectra using a least-squares minimized $\chi^{2}$ fitting routine. We used atomic data from Bely-Dubau et al. (1982) with slight modifications to improve the fits (Sterling et al. 1997). We also used ionization equilibrium data of Arnaud \& Rothenflug (1985), and we assumed a calcium abundance of $4.5 \times 10^{-6}$ relative to $\mathrm{H}$. We accumulated data until the total number of counts exceeded 10,000 in each spectrum, resulting in accumulation times ranging from about $6 \mathrm{~s}$ to $300 \mathrm{~s}$, with only the three earliest spectra exceeding $100 \mathrm{~s}$ accumulation time. Accurate fits to spectra from early in the development of flares near solar disk center often require the addition of a second, blue-shifted component, which appears to represent upflowing plasmas on vertical flux tubes toward Earth (e.g., Antonucci et al. 1982; Fludra et al. 1989; Doschek 1990; Mariska 1994). For this event, however, the spectra are largely symmetric, and no such blue-shifted component is required. This indicates that the material flows are likely to be substantially away from vertical in this flare, which corroborates our suspicions from EIT and SXT images and MDI magnetograms that the flaring loops are pushed away from vertical by the sunspot fields (e.g., Fig. 2).

From the fits to the spectra we derive fluxes, electron temperature $\left(T_{e}\right)$ and emission measure (EM) (e.g., Sterling et al. 1997). Figure 8 shows these derived quantities for the 1998 May 1 event. Comparison with Figure 7 shows an increase in $T_{e}$ beginning from the earliest spectrum, but remaining relatively flat between 22:30 UT and 22:40 UT; this corresponds to the period over which region $\mathrm{D}$ in Figure $6 \mathrm{i}$ brightens. A substantial jump in $T_{e}$ coincides with the brightenings of regions $\mathrm{A}$ and $\mathrm{B}$, and the hard $\mathrm{X}$-ray bursts in Figures $7 \mathrm{~b}$ and $7 \mathrm{c}$.

\section{Event of 05 UT, 1998 May 2}

We next consider the May 2 event peaking near 5 UT. Figure 9 shows SXT full-resolution partial-frame images at four different times. Figures $9 \mathrm{a}$ and $9 \mathrm{~b}$ are from near the start of the 
eruption, faintly showing extensions from the core region to the southeast, similar to Figure $2 \mathrm{~b}$ for the May 1 event. Figure 9c shows a reduced field-of-view image near the time of peak soft X-ray intensity, and Figure 9f shows post-flare loops developing in the region. Temporally, Figures 9a, 9c, and $9 \mathrm{f}$ closely correspond to the EIT images in Figures $4 \mathrm{~b}, 4 \mathrm{c}$, and 4e, respectively. Figure 9d shows the same image as that in Figure 9c, overlaid with the same magnetogram used in Figure 2, and a hard X-ray image synthesized from HXT's M2-band. Morphologically, this event is very different from the earlier event, with more compact soft X-ray brightenings and a single, strong hard X-ray source. Figure 9e shows boxes around two regions which display dynamic intensity changes, as well as a box around the entire flaring region.

Figure 10 shows the lightcurves from the regions boxed in Figure 9e, along with HXT and BATSE hard X-ray lightcurves. As in the event of May 1, lightcurves from different regions show distinct characteristics: Region B begins brightening near the time of EIT crinkle formation (Fig. 4b), well before the hard X-ray burst, while region A shows a sharp increase in intensity coincident with the hard X-ray burst. This case differs, however, from the May 1 event in key aspects. First, the early-brightening region is much more intense in soft X-rays than the region that brightens with the hard X-rays in this case, whereas the intensities were much more comparable in the May 1 case. Second, there is a single principal hard X-ray source in this case, and its location coincides (to within our resolution limits) with the location of the earliest soft X-ray brightening of Box B of Fig. 9e, and not with the later soft X-ray brightening of Box A, even though it is the lightcurve of Box A which responds most acutely to the hard X-ray burst. A third key difference between this case and the May 1 event is that the hard X-ray spectrum is much harder in this case; the intensities from the L, M1, and M2 HXT bands are approximately equal in this May 2 event (for clarity the M1 and M2 intensities are scaled factors of 0.5 and 0.2, respectively, in Fig. 10b), whereas the L-band was much stronger than the M1-band in the May 1 event (M1 is scaled by a factor of 0.5 in Fig. 7b). Similarly, the intensity of the harder BATSE channel is only slightly less than that of the softer BATSE channel in Figure 10c, in contrast to the May 1 event (Fig. 7c) where the difference is more pronounced (the higher-energy BATSE channel intensity is scaled by a factor of 1.0 and 0.5 in Figs. 7c and 10c, respectively).

BCS Ca XIX results for the May 2 event, given in Figure 11, show features similar to those of the May 1 event. Prior to the hard X-ray burst, the spectra reflect a plasma with elevated, but roughly constant, $T_{e}$. Roughly coincident with the hard X-ray burst, $T_{e}$ starts to rise. Similar to the May 1 event, we found that single-component fits are adequate for fitting spectra for this event. That is, there is very little or no evidence for a blue-shifted component, suggesting that most mass flows normal to the Sun-Earth line-of-sight, again consistent with the event-associated loops being pushed away from the vertical by the sunspot fields.

\section{Discussion}

In EIT images, the two flares we examined appear very similar: Dynamic EIT crinkles form remotely from the core of the erupting region prior to substantial brightenings in the core, post-flare loops develop starting in the core region, and the process eventually repeats. Also, soft X-ray lightcurves from one region of each flare show an increase prior to the hard X-ray burst, while other regions show a sharp intensity increase coincident with the hard X-ray burst. We also found, however, that the core region of the two flares appear very different, with widely-separated soft X-ray brightenings of similar intensity and well-separated hard X-ray sources with a soft hard-X-ray 
spectrum in the May 1 event, and more-compact principal soft X-ray brightenings and a single hard X-ray source with a harder hard X-ray spectrum in the May 2 event. BCS spectra show similarities in the two events: heating prior to the hard X-ray burst which results in an approximately constant $T_{e}$, followed by an impulsive surge in $T_{e}$ associated with the hard X-ray burst. Neither event shows strong blueshifts, consistent with mass motions occurring normal to the line-of-sight. Thus the flares repeat with similar patterns in some aspects, but other aspects differ between the two flares.

We can reconsider the schematic of Figure 5 in light of the common elements of our new observations. Sterling \& Moore (2001) suggested that each flaring episode began when a portion of the flux rope in the core erupts and its envelope reconnects (external reconnection) with coronal hole fields. This external reconnection led to the formation of the EIT crinkles and modifications to the boundaries of the coronal hole. Our new investigations, however, show indications of EIT crinkle formation prior to discernible activity in the core region in soft X-rays. For example, for the May 1 event Figure 3 shows EIT crinkles definitely forming by 22:05 UT, and maybe as early as 21:48 UT, whereas Figure 7a shows the first indication of significant increase in soft X-ray intensity in the core near 22:15 UT. There are also no substantial hard X-rays detected until well after the EIT crinkle formation, although it is unclear whether, e.g., early-phase magnetic reconnection in the core would be expected to be strong enough to generate hard X-rays. A loop feature in the core that may correspond to the brightening in soft X-rays in box D of Figure 6i does become visible in EIT just after EIT crinkle onset; arrows point out this features in soft X-rays in Figure $6 \mathrm{~h}$, and in EUV in Figure 3c. This feature is not obvious in the previous EIT image in Figure 3b, when EIT crinkle-associated activity appears to start. Thus there is no indication in SXT or EIT of activity in the core preceding the EIT crinkles.

It is not possible to determine with certainty the ordering between the EIT crinkles and the SXT core brightenings in the May 2 event, since Figure 4 shows EIT crinkle formation beginning around 4:36 UT while the earliest SXT data (which show an intensity rise in progress following Yohkoh spacecraft night) are at about 4:38 UT in Figure 10a. Figure 1, however, shows that the GOES soft X-ray intensity does not begin to rise any earlier than 4:32 UT, and so the GOES soft X-ray flux rise does not precede the appearance of the EIT crinkles by more than 4 minutes at most. (As noted at the end of $\S 2$, the GOES C1 event peaking near 04:35 UT on May 2 occurs in a different active region, but it may mask the start of the intensity rise of the AR 8210 event.) In EIT images, however, EIT crinkles begin to develop prior to any noticeable change in the core region. Figure 4b shows an EIT crinkle beginning in the southeast, while the core is virtually identical in Figures $4 \mathrm{a}$ and $4 \mathrm{~b}$. In contrast, there is a definite brightening in the core in Figure 4c, when the EIT crinkles are well-developed. Our results, therefore, indicate that external reconnection producing the EIT crinkles begins prior to significant disruption of the core fields in both events, but the delay between the EIT crinkles and the core disruption is greater in the May 1 event. Our new observations do not alter our view of the subsequent progression of events outlined in the schematic and associated caption of Figure 5.

As noted in $\S 2$, the start of the first major event of the homologous sequence (occurring at 17:57 UT May 1) occurs during Yohkoh night. The available SXT images of that event, however, are similar to those of the May 1 event. Also, EIT images for that event show indications of EIT crinkles prior to a clear indication of a disruption to the core. Therefore, the data we have for this event are consistent with the conclusions drawn from our May 1 and May 2 events regarding timings of EIT crinkles and disruption of the core.

These observations have implications for the energy-release mechanism for the eruption. As 
noted in $\S 1$, two suggested mechanisms are tether cutting and the breakout model. Tether cutting suggests that reconnection in the core is the source of the eruption release. At first, the heating rate from this reconnection would be small, gradually increasing as the reconnection proceeds. Quantitative estimates for the expected energy-release-rate growth are not available, and hence we cannot say how much of a delay there should be between the onset of the tether cutting and the first core brightenings in soft X-rays. Even so, it seems unlikely that the formation of EIT crinkles and the associated formation of hot coronal loops linking the core region to the EIT crinkle locations should precede EUV and soft X-ray brightenings in the core if the external reconnection is driven by expulsion of the core unleashed by internal tether cutting. With the breakout model, however, this is just the ordering that would be expected: Reconnection far from the core region between restraining fields - in this case the combined fields of the sunspot and the coronal hole - and the fields of the EFR would lead to a breach in the overlying fields in the vicinity of a magnetic null point. Our geometry is somewhat different from that for breakout presented by Antiochos et al. (1999), but the principle is the same. In our case, the ultimate driver of the external reconnection would be the buoyancy causing the EFR to emerge. Growth of the EFR leads to the external reconnection, which powers the EIT crinkles and brightening of the associated soft X-ray loops extending from the core region to the EIT crinkle locations. Here we have assumed that enough stress can build up in the vicinity of the null point in the geometry of Figure 5a to result in the external reconnection; we can only say that this assumption is consistent with our observations. Future numerical simulations should be able to address this assumption from a theoretical standpoint.

Applying the concepts introduced by Antiochos (1998) to our case, only after the breach in the overlying fields grows large enough do the EFR fields accelerate outward, leading to internal reconnection in the vicinity of the core, and the associated soft and hard X-ray brightenings in the core region. Continued emergence of flux of the EFR would provide a natural driver for the repeated eruptions in the homologous sequence. Moore et al. (2001) presented evidence that eruptions can occur in seemingly bipolar field regions, which is consistent with the tether cutting model but does not fit the breakout picture. While the Moore et al. (2001) observations were of events with a particularly simple geometry, SXT images of the erupting core region here are too complex to identify loops possibly involved in tether cutting. If tether cutting does operate here and begins earlier than the EIT crinkles, we have not found evidence for it in coronal emission. We can only conclude that our findings for the events studied in this paper are consistent with the breakout model for eruptions. It may be that tether cutting plays a major role in initiation of the eruption only in a subset of flares, and breakout plays a major role in a different subset of flares. We recognize, however, that another possibility is that even seemingly bipolar regions may be only the most obvious portions of a breakout-type of geometry. In that case, local magnetic circumstances would be responsible for a more comprehensive view of the breakout eruption topology being observable in some cases, such as those considered here, while only the bipolar core region is prominent in other cases.

Aulanier et al. (2000) also observed a flare in a multi-polar, delta-spot region which they argue is compatible with the breakout model. Using TRACE data, they detect preflare motions of loops in EUV beginning about more than an hour prior to flare maximum. This is consistent with our observations of EIT crinkles $\approx 40-50$ min prior to flare maximum for the May 1 and May 2 events. EIT data are not available for the time of the Aulanier et al. (2000) flare, and so a direct comparison with EIT images is not possible for that case. Comparisons in other cases where data from both instruments are available will be useful for determining whether the motions seen by Aulanier et al. (2000) are high-resolution manifestations of the EIT crinkles. 
As noted in $§ 1$, Sterling \& Moore (2001) estimated the velocity of the outward-expanding and inward-fading EIT crinkles to be $\sim 20 \mathrm{~km} \mathrm{~s}^{-1}$. These values, however, were based on rather ill-defined EIT crinkle emissions, and furthermore, there is considerable freedom in interpretation of the velocities (Sterling \& Moore 2001). Therefore, we do not believe the velocities are appropriate for using as a discriminator for accepting or rejecting the breakout model with this data set.

Our BCS results in Figures 8 and 11 allow us to estimate the energy lost to radiation during the eruptions. Energy losses due to radiation are given by

$$
l=n_{e}^{2} \Phi\left(T_{e}\right)
$$

erg $\mathrm{cm}^{-3} \mathrm{~s}^{-1}$, where $n_{e}$ is the electron density and $\Phi$ is the radiative loss function. Approximating the emission measure by

$$
E M=\int n_{e}^{2} d V \approx n_{e}^{2} V
$$

where $V$ is the emitting volume, we have a loss rate of

$$
L \approx E M \times \Phi\left(T_{e}\right)
$$

$\mathrm{erg} \mathrm{s}^{-1}$. We can calculate this energy during the pre-hard X-ray burst time period for the May 1 event. From Figure 9, between 22:30 UT and 22:40 UT, $T_{e}$ is approximately constant at just over $10 \mathrm{MK}$, which corresponds to $\Phi \approx 4 \times 10^{-22} \mathrm{erg} \mathrm{s}^{-1} \mathrm{~cm}^{-3}$ (Raymond \& Smith 1977) while $E M \sim 10^{48} \mathrm{~cm}^{-3}$. This yields $L \sim 5 \times 10^{26} \mathrm{erg} \mathrm{s}^{-1}$, or $L_{t o t} \approx 3 \times 10^{29}$ erg over the ten minute period. These values correspond (to order-of-magnitude) to the amount of energy radiated in the core-field region over the time which the EIT crinkles are evolving. Near the peak of the flare at about 22:48 UT, the temperature is only slightly higher, while $E M$ increases by an order-of-magnitude, increasing the radiative energy output by an order-of-magnitude via equation (4). Numbers for the May 2 event are similar for the pre-peak phase, but the $E M$ only reaches about half the value of that of the May 1 flare during its peak.

Since the soft X-ray signal from the faint loops connecting the core to the EIT crinkles (e.g., Fig. 2b) is substantially smaller than the emission from the core during times of the EIT crinkle development, and since the temperature of those loops are likely to be less than those of the core, our energy rate for the core plasmas prior to the hard X-ray burst should be an upper limit on the energy released in these loops. Consequently, the energy expended due to the external reconnection resulting in these loops and EIT crinkles will be $\lesssim 10^{26} \mathrm{erg} \mathrm{s}^{-1}$. Thus the overlying fields would have to play a passive role of inhibiting the much-more-energetic core eruption, rather than driving the eruption itself; this is consistent with the breakout picture.

For both of our events we find a correlation between $T_{e}$ and hard X-rays; such a correlation was also noted in a larger sample of events by Sterling et al. (1997). Since the pre-hard X-ray-burst soft X-ray source and the soft X-ray sources that respond to the hard X-ray burst are spatially different in the May 1 case, this latter correlation suggests that plasma in different loops may have different temperature structures. Although the May 2 images indicate that the hard X-ray and principal soft X-ray sources may originate from the same location, this may be a consequence of insufficient resolution, since, as pointed out by Warren (2000), fine structure may exist in high-temperature portions of solar flares.

We thank W. Henze and R. Schwartz for BATSE data and instructions on their reduction. We also thank H. S. Hudson for motivating discussions and valuable comments on the manuscript, and 
we thank B. Hargrave for assistance with computer and video equipment. ACS held a National Research Council-NASA/MSFC Research Associateship. RLM was supported by funding from NASA's Office of Space Science through the Solar Physics Supporting Research and Technology Program and the Sun-Earth Connection Guest Investigator Program. SOHO is a project of international cooperation between ESA and NASA, and Yohkoh is a mission of the Institute of Space and Astronautical Sciences (Japan), with participation from the U. S. and U. K. 


\section{REFERENCES}

Antiochos, S. K. 1998, ApJ, 502, L181

Antiochos, S. K., DeVore, C. R., \& Klimchuk, J. A. 1999, ApJ, 510, 485

Antonucci, E., et al. 1982, Solar Phys., 78, 107

Aulanier, G., DeLuca, E. E., Antiochos, S. K., McMullen, R. A., \& Golub, L. 2000, ApJ, 540, 1126

Arnaud \& Rothenflug, R. 1985, A\&AS, 60, 425

Bely-Dubau F., et al. 1982, MNRAS, 201, 1155

Berger, T. E., De Pontieu, B., Schrijver, C. J., \& Title, A. M. 1999, ApJ, 519, L97

Canfield, R. C., Hudson, H. S., McKenzie, D. E. 1999, Geophys. Res. Lett., 26, 627

Culhane, J. L., et al. 1991, Solar Phys., 136, 89

Delaboudiniere, J.-P. et al. 1995, Solar Phys., 162, 291

Doschek, G. A. 1990, ApJS, 73, 117

Fishman, G. J., et al. 1989, in Proceedings of the Gamma Ray Observatory Science Workshop, ed. W. N. Johnson, (Greenbelt: GSFC), 2

Fletcher, L. \& De Pontieu, B. 1999, ApJ, 520, L135

Fludra, A., Lemen, J. R., Jakimiec, J., Bentley, R. D., \& Sylwester, J. 1989, ApJ, 344, 991

Glover, A., Ranns, N. D. R., Harra, L. K., \& Culhane, J. L. 2000, Geophys. Res. Lett., in press

Howard, R. A., Michels, D. J., Sheeley, N. R., \& Koomen, M. J. 1982, ApJ, 263, L101

Hirayama, T. 1964, Solar Phys., 34, 323

Hudson, H. S., Lemen, J. R., St. Cyr, O. C., Sterling, A. C., \& Webb, D. F. 1998, Geophys. Res. Lett., 25, no. 14, 2481

Kahler, S. 1977, ApJ, 214, 891

Kosugi, T., et al. 1991, Solar Phys., 136, 17

Mariska, J. T. 1994, ApJ, 434, 756

Martens, P. C. H., Kankelborg, C. C., \& Berger, T. E. 2000, ApJ, 537, 471

Moore, R. L., and LaBonte, B. 1980, in Proc. Symp. on Solar and Interplanetary Dynamics, Reidel, Boston, 207

Moore, R. L., Schmieder, B., Hathaway, D. H., \& Tarbell, T. D. 1997, Solar Phys., 176, 153

Moore, R. L., Sterling, A. C., Hudson, H. S., \& Lemen, J. R. 2001, ApJ, in press

Raymond, C. \& Smith, B. W. 1977, ApJS, 35, 419

Shibata, K., Nitta, N., Strong, K. T., Matsumoto, R., Yokoyama, T., Hirayama, T., Hudson, H., \& Ogawara, Y. 1994, ApJ, 431, L51

Sterling, A. C., Hudson, H. S., Lemen, J. R., \& Zarro, D. A. 1997, ApJS, 110, 115

Sterling, A. C. 2000, J. of Atmos. and Solar-Terrestrial Phys., in press

Sterling, A. C., Hudson, H. S., Thompson, B. J., \& Zarro, D. M. 2000, ApJ, 532, 628

Sterling, A. C., \& Moore, R. L. 2001, JGR, in press

Sturrock, P. A. 1989, Solar Phys., 121, 387 
Tang, F., \& Moore, R. L. 1982, Solar Phys., 77, 263

Tsuneta, S., et al. 1991, Sol. Phys., 136, 37

Warren, H. P. 2000, ApJ, 536, L105

Webb, D. F., Cliver, E. W., Crooker, N. U., St. Cyr, O. C., Thompson, B. J. 2000, J. Geophys. Res., 105,7491

This manuscript was prepared with the AAS LATEX macros v4.0. 
Fig. 1. - Soft X-ray profiles for the 1998 May 1 and May 2 events of this study from the GOES 9 spacecraft. Hashed areas represent times of Yohkoh spacecraft night. In each panel, upper curves are from the $1-8 \AA$ channel and lower curves are from the $0.5-4 \AA$ channel. Vertical axis units are $\mathrm{W} \mathrm{m} \mathrm{m}^{-2}$.

Fig. 2.- Views of AR 8210 before (a, c, and e) and near the time of (b, d, and f) the start of the 1998 May 1 flare's eruption. (a) and (b) are SXT AlMg and Al1 filter images, respectively, and (c) and (d) are EIT $195 \AA$ filter images. A magnetogram taken at 22:24 UT on 1998 May 1 from the SOHO MDI instrument overlays the images in (a) and (b), with contour levels of 25, 40, and $500 \mathrm{G}$, and with yellow and red indicating negative and positive polarities, respectively. Panels (e) and (f) show this same magnetogram overlaid on the EIT images of panels (c) and (d), respectively. Arrows in (f) indicate examples of EIT crinkles. North is up and west is to the right, and size scales are in arcseconds measured from disk center.

Fig. 3.- Sequence of EIT $195 \AA$ filter images showing evolution of the May 1 flare. The arrow in the lower-left in (c) points to the same EIT crinkle indicated by the arrow in the lower-left of Fig. 2f, while the arrow in (b) points to a possible early phase of this same EIT crinkle. The arrow in (d) points to an area of EIT crinkles in the northwest. The arrow in the center of (c) points to a possible EUV manifestation of a core-region feature prominent in soft X-rays (Fig. 6h). The image in Figs. (2c) and (2e) occurs prior to (a) and the image in Figs. (2d) and (2f) occurs in-between (c) and (d). Postflare loops appear in (e) and (f). North is up and west is to the right, and size scales are in arcseconds measured from disk center.

Fig. 4.- Same as Fig. 3 for the next flare in the homologous sequence. Arrows indicate EIT crinkles in (b) - (d), and postflare loops appear in (e) and (f). North is up and west is to the right, and size scales are in arcseconds measured from disk center.

Fig. 5.- Schematic diagram depicting the suspected progression of events seen in Figs. 3 and 4, as described in Sterling and Moore (2000). Lines represent magnetic field lines linking negative (pointing downward) or positive (pointing upward) polarities. Dashed lines indicate new field lines about to be formed in a reconnection episode about to take place. To aid in clarity, some field lines are bold (closed field lines and flux-rope field lines), while others are thin (open fields and sunspot field lines). (a) A flux rope is lodged beneath an EFR, appearing as a sigmoid in soft X-rays. (b) The envelope of an erupting portion of the flux rope reconnects (external reconnection) with coronal hole fields, increasing the extent of the anemone lobe beneath it and producing EIT crinkles at the feet of the lobe. A lower portion of the flux rope remains at the original location and continues to emerge. Internal reconnection also occurs, augmenting the flux wrapping the flux rope, and causing a flare and postflare loops below via the standard reconnection flare model. (c) Virtually all of the coronal hole magnetic field has been consumed by external reconnection. The erupting wrapped flux rope is about to escape as a CME, leaving a flare and growing postflare loops in its wake. (d) Reconnection (external reconnection) between the anemone lobe fields and the newly-reconnected open fields (i.e., fields like the uppermost dashed line in b) lead to a reformation of the coronal hole and the retraction of the anemone fields with retracting EIT crinkles at the base. After some time the situation in (a) is restored, and after further emergence a new eruption will restart the cycle. In (b) and (d), clear rectangles indicate locations of ongoing external reconnection, and in (b) and (c) shaded rectangles indicate regions of the ongoing internal reconnections. Most energy is released in the internal reconnections, which result in the bulk of the soft and hard X-ray flare emissions.

Fig. 6. - SXT images of AR 8210 during the May 1 event associated with the EIT crinkles of Figs. 2 
and 3. These are full-resolution $\left(2.455^{\prime \prime}\right.$ pixels $)$ partial frame images $(\mathrm{a}-\mathrm{j})$, or half-resolution $\left(4.910^{\prime \prime}\right.$ pixels) full-disk images ( $\mathrm{k}$ and $\mathrm{l})$. The image in $(\mathrm{k})$ is from the AlMg filter, while all other images are from the Al.1 filter. Panels (g) - (i) are identical, with a $19.5 \mathrm{~s}$ duration HXT low channel (13.9$22.7 \mathrm{keV}$ ) image from 22:45:34 UT overlaid as contours (at 50\% and $90 \%$ of maximum intensity) in (h), and boxes corresponding to the lightcurves in Fig. 7a in (i). All images are scaled logarithmically, except for (l), which is scaled linearly due to its low intensity. Increases in SXT intensity occur in the core region after the time of formation of the earliest EIT crinkles in Fig. 3. The arrow in (h) points out a loop feature possibly corresponding to one seen in EUV (Fig. 3c). North is up and west is to the right, and size scales are in arcseconds measured from disk center.

Fig. 7.- (a) Lightcurves from regions marked in the SXT image in Fig. 6i, showing the average intensity per box (i.e., each point on a given lightcurve represents the sum of the intensity in the respective box divided by the exposure time and the number of pixels in that box). (b) Hard X-ray fluxes from the HXT L-band (upper plot; $13.9-22.7 \mathrm{keV}$ ) and M1 band (lower plot; $22.7-32.7 \mathrm{keV}$ ); for clarity, the M1 flux is plotted a factor of 2 lower than its actual value. (c) Hard X-ray fluxes from BATSE's 25-50 keV (upper plot) and 50-100 keV (lower plot) channels (no scale-reduction factor is used in this case). BATSE data earlier than 22:24 UT are not available due to spacecraft night.

Fig. 8.- (a) Thick line: BCS flux from Ca XIX spectra, with scale on left-side axis. Thin line: SXT flux from the total flaring region (box C in Fig. 6i, and the thin dashed line in Fig. 7a), with scale on the right-side axis. (b) Thick line: electron temperature derived from BCS Ca XIX spectra, with scale on left-side axis. Uncertainties in temperature due to Poisson statistics are typically $0.5-1.5 \mathrm{MK}$. Thin line: emission measure obtained from BCS Ca XIX spectra, with scale on right-side axis.

Fig. 9.- SXT images of AR 8210 during the event associated with the EIT crinkles of Fig. 4 ("May 2 event"). All images are logarithmically scaled, full-resolution (2.455" pixels) partial frame images taken with the Al.1 filter. Panels (c) - (e) are identical, with the magnetogram of Fig. 2 overlaid and with an HXT M2 channel $(32.7-52.7 \mathrm{keV})$ image overlaid as the black contours in (d) at 50 and $90 \%$ of maximum intensity. There is only one hard X-ray source, highly concentrated and located near $\mathrm{X}=150$ and $\mathrm{Y}=-270$. Boxes in (e) correspond to the lightcurves in Fig. 10a. North is up and west is to the right, and size scales are in arcseconds measured from disk center.

Fig. 10.- (a) Lightcurves from regions marked in the SXT image in Fig. 9e, showing the average intensity per box. (b) Hard X-ray fluxes from the HXT L-band (upper plot; $13.9-22.7 \mathrm{keV}$ ), M1 band (middle plot; 22.7-32.7 keV), and M2 band (lowest plot; $32.7-52.7 \mathrm{keV}$ ). For clarity, the M1 and M2 fluxes are plotted factors of 2 and 5, respectively, lower than their actual values; if plotted without reduction, the three curves would nearly overlap, indicating a much harder hard X-ray spectrum than that of the event in Fig. 7. (c) Hard X-ray fluxes from BATSE's $25-50 \mathrm{keV}$ (upper plot) and 50-100 keV (lower plot) channels; the 50-100 keV intensity is reduced by a factor of 2 for clarity. BATSE data earlier and later than those plotted are not available over the time range considered.

Fig. 11.- (a) Thick line: BCS flux from Ca XIX spectra for the May 2 event. Thin line: SXT flux from the total flaring region (box $\mathrm{C}$ in Fig. 9e, and the thin dashed line in Fig. 10a). (b) Thick line: Electron temperature derived from BCS Ca XIX spectra, with scale on left-side axis. Uncertainties in temperature due to Poisson statistics are typically $0.4-0.8 \mathrm{MK}$. Thin line: emission measure obtained from BCS Ca XIX spectra, with scale on right-side axis. 

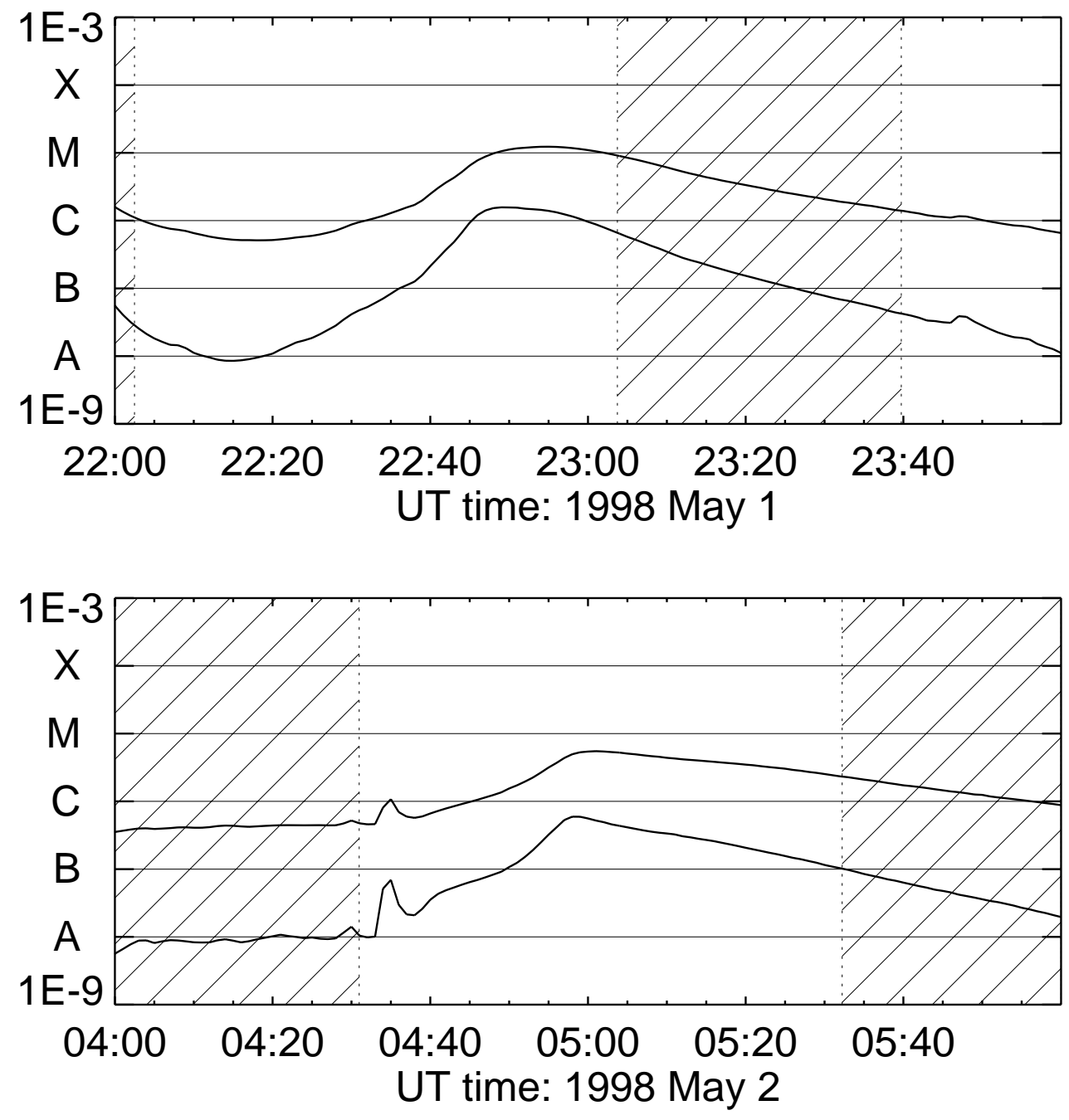

Fig. 1.- 
(a) SXT: 1-May-1998 21:12:51 UT

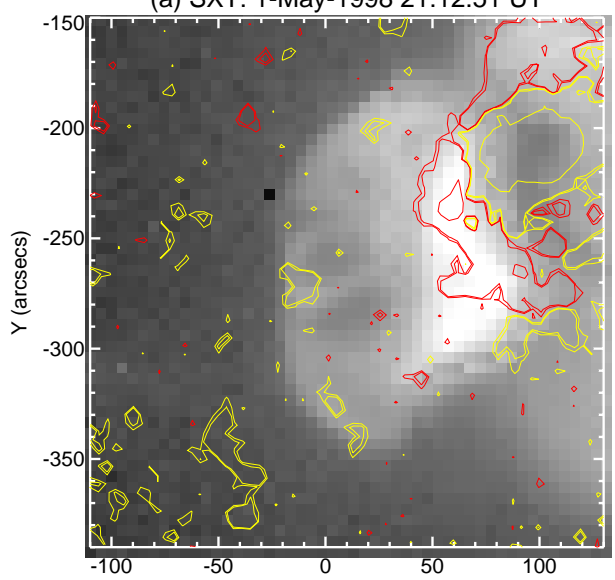

(c) EIT: 1-May-1998 21:14:05 UT

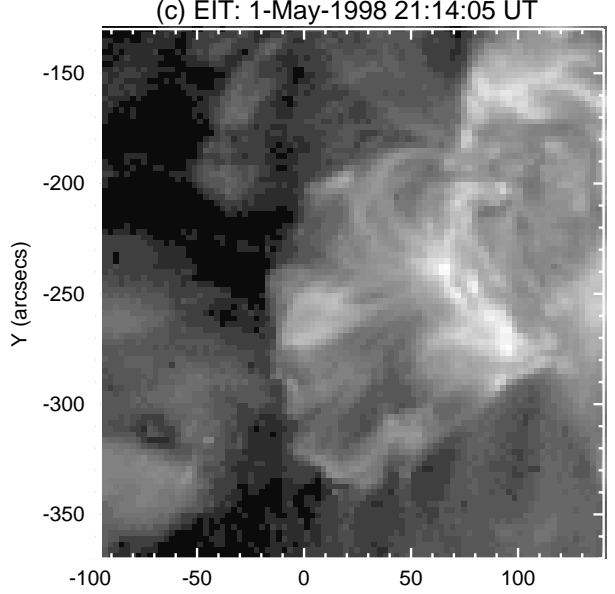

(e) EIT: 1-May-1998 21:14:05 UT

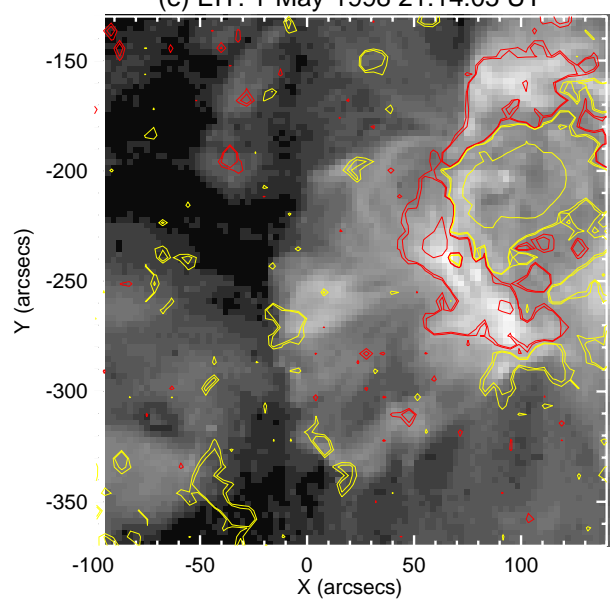

(b) SXT: 1-May-1998 22:16:49 UT

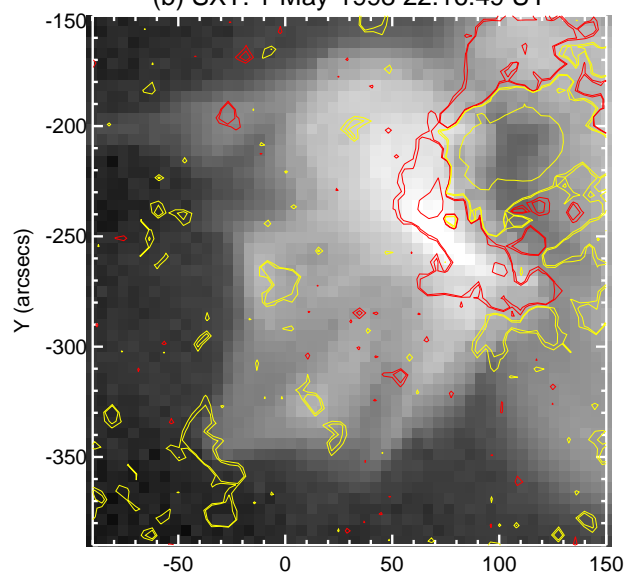

(d) EIT: 1-May-1998 22:22:53 UT

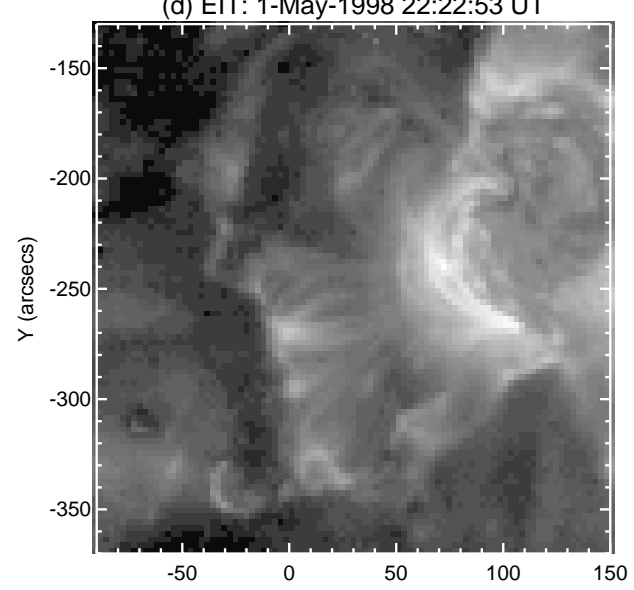

(f) EIT: 1-May-1998 22:22:53 UT

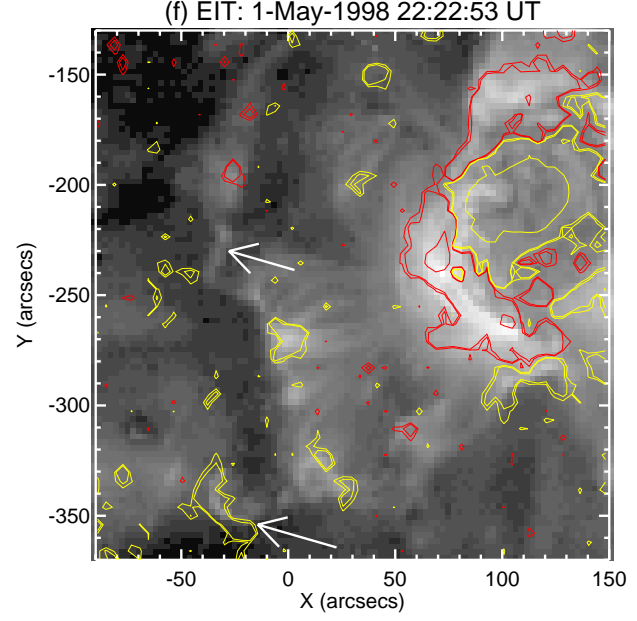

Fig. 2.- 

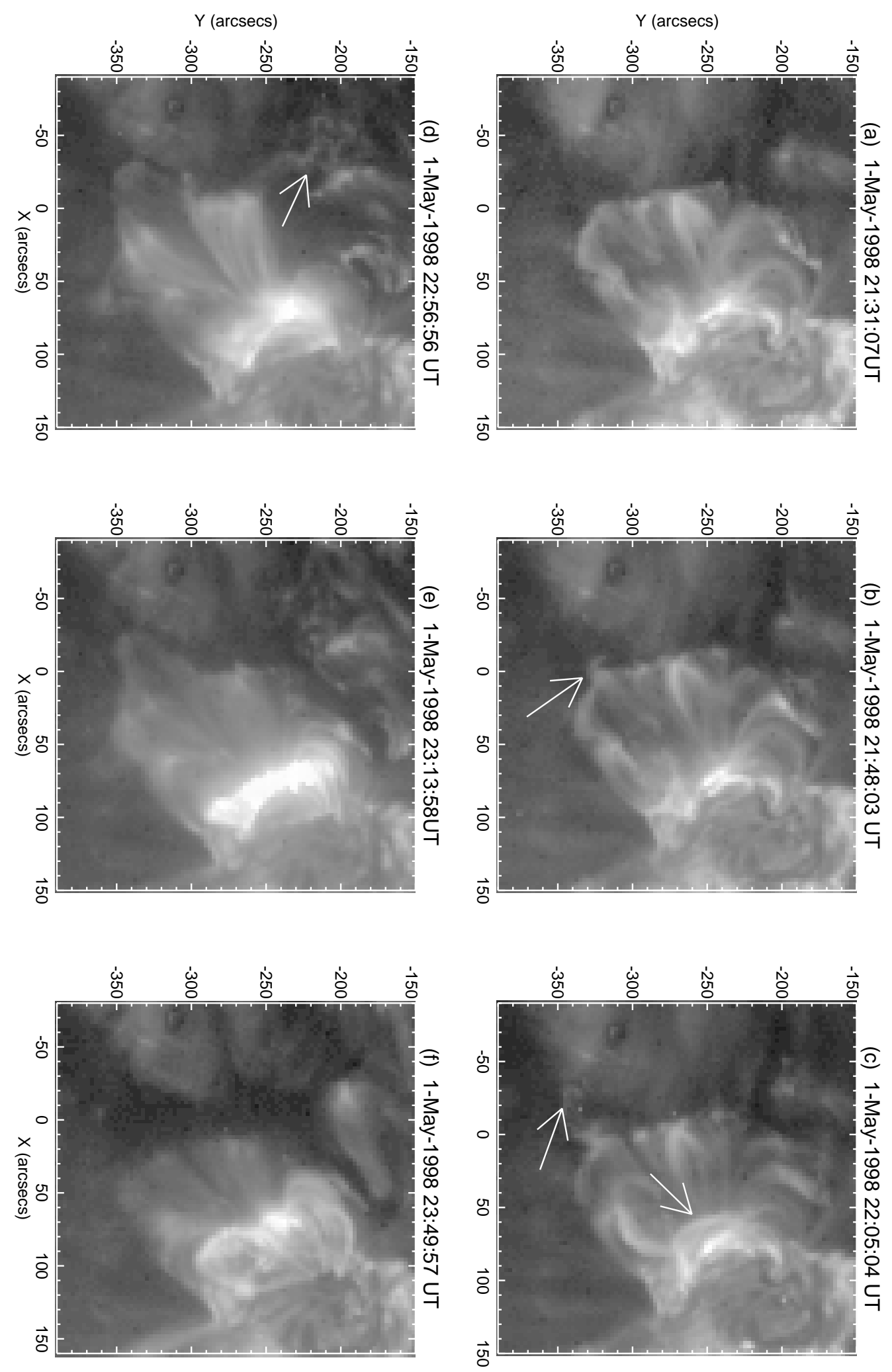

Fig. 3.- 

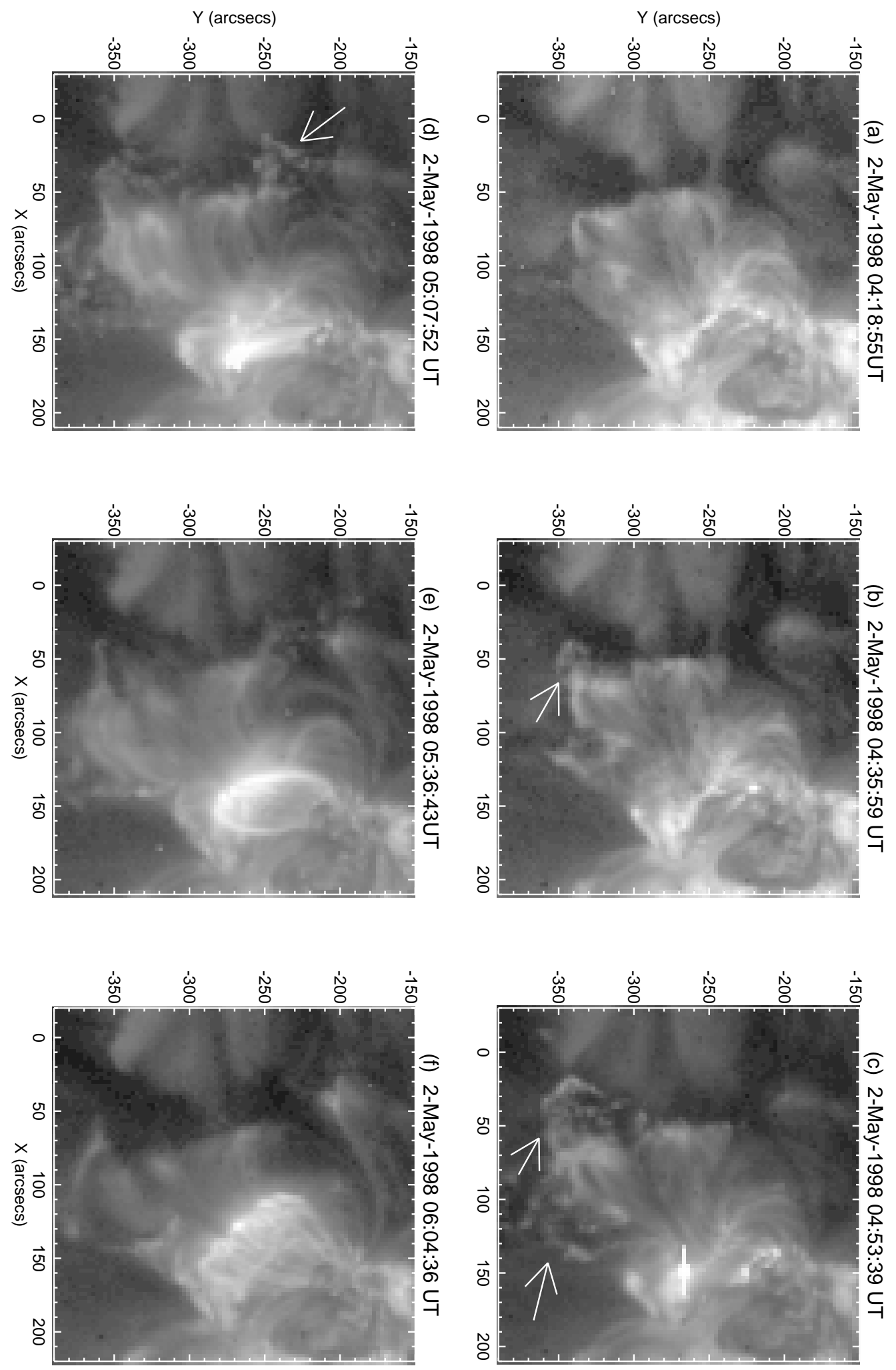

Fig. 4.- 

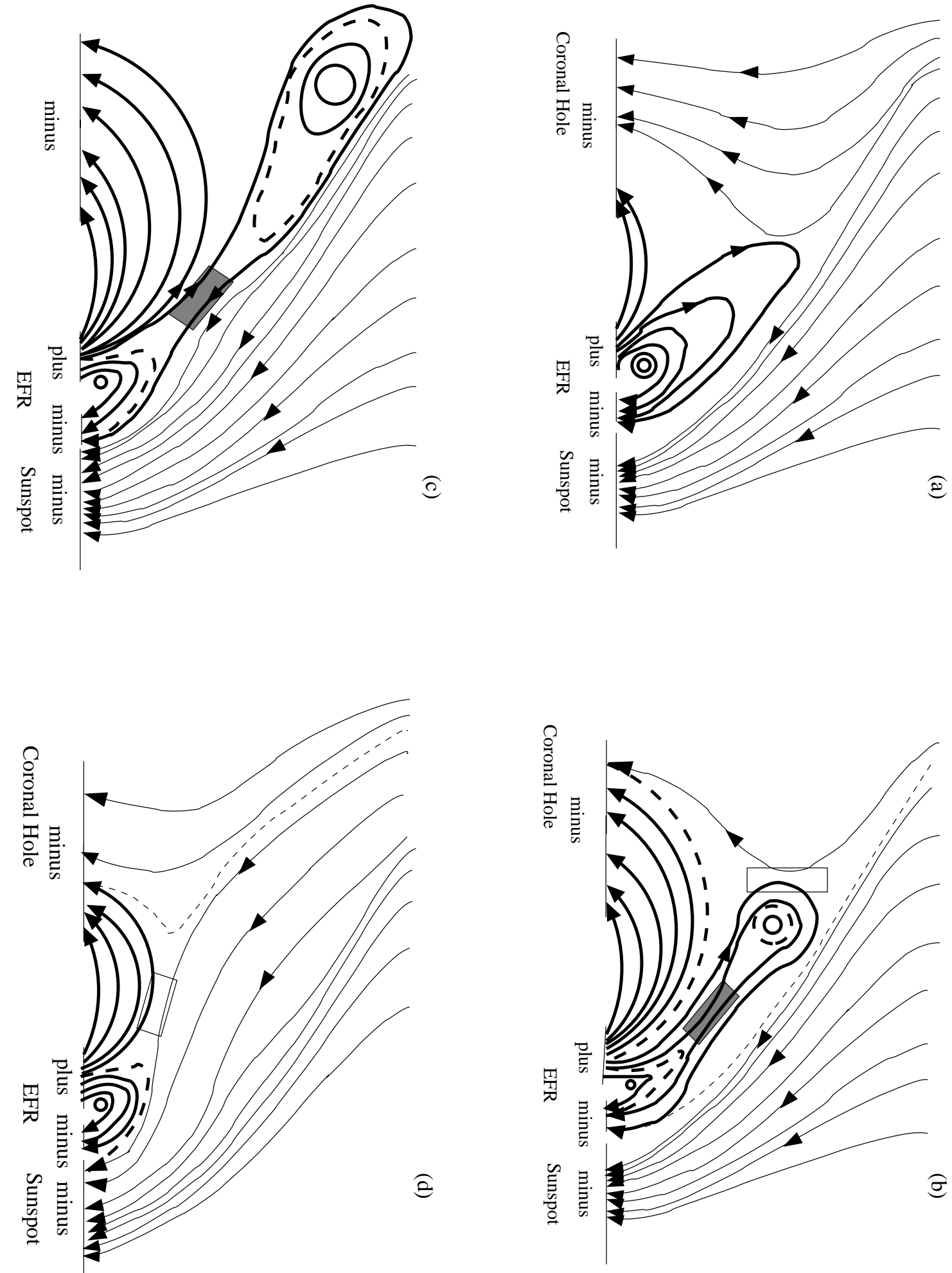

Fig. 5.- 

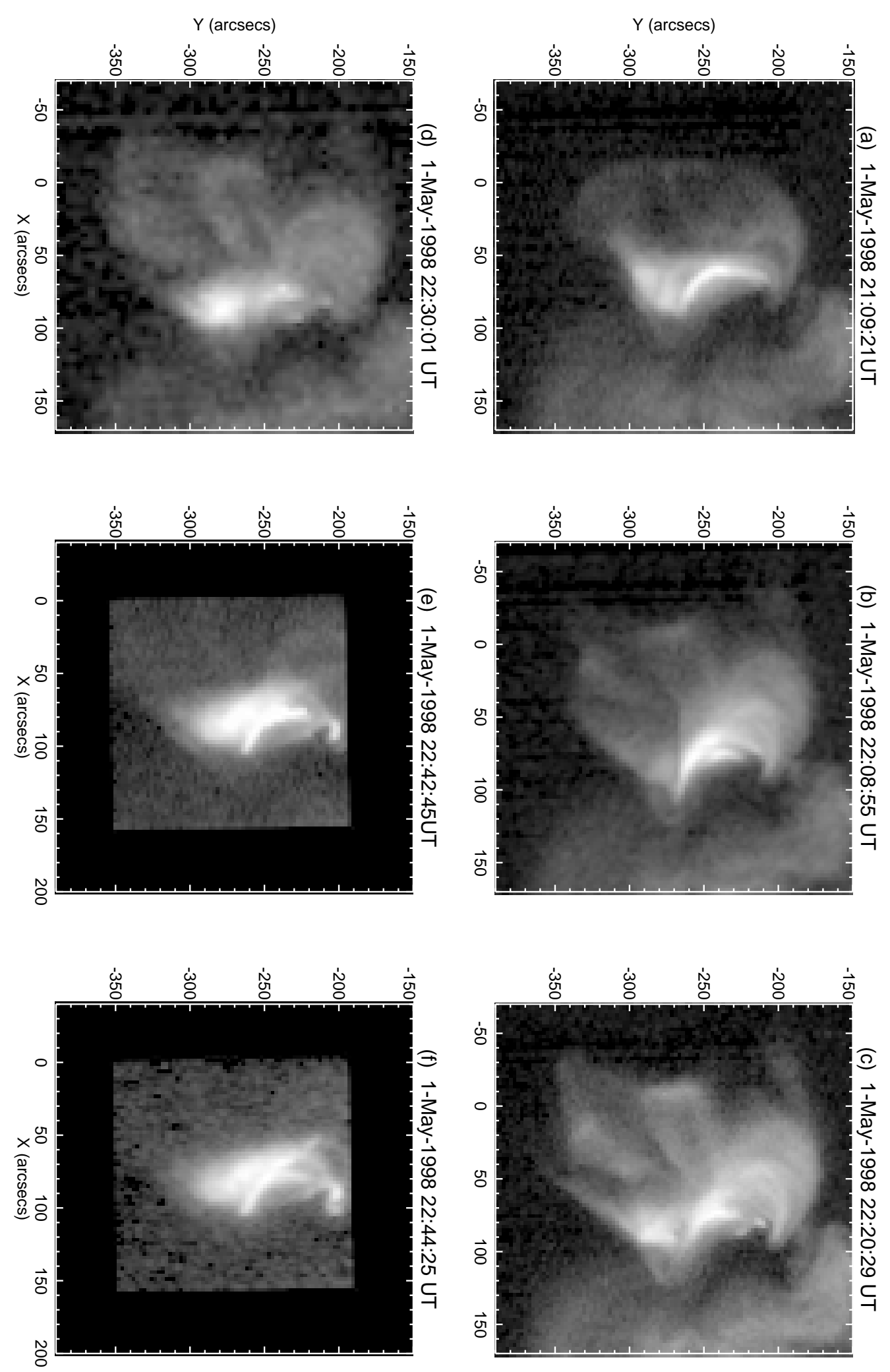

Fig. 6.- 

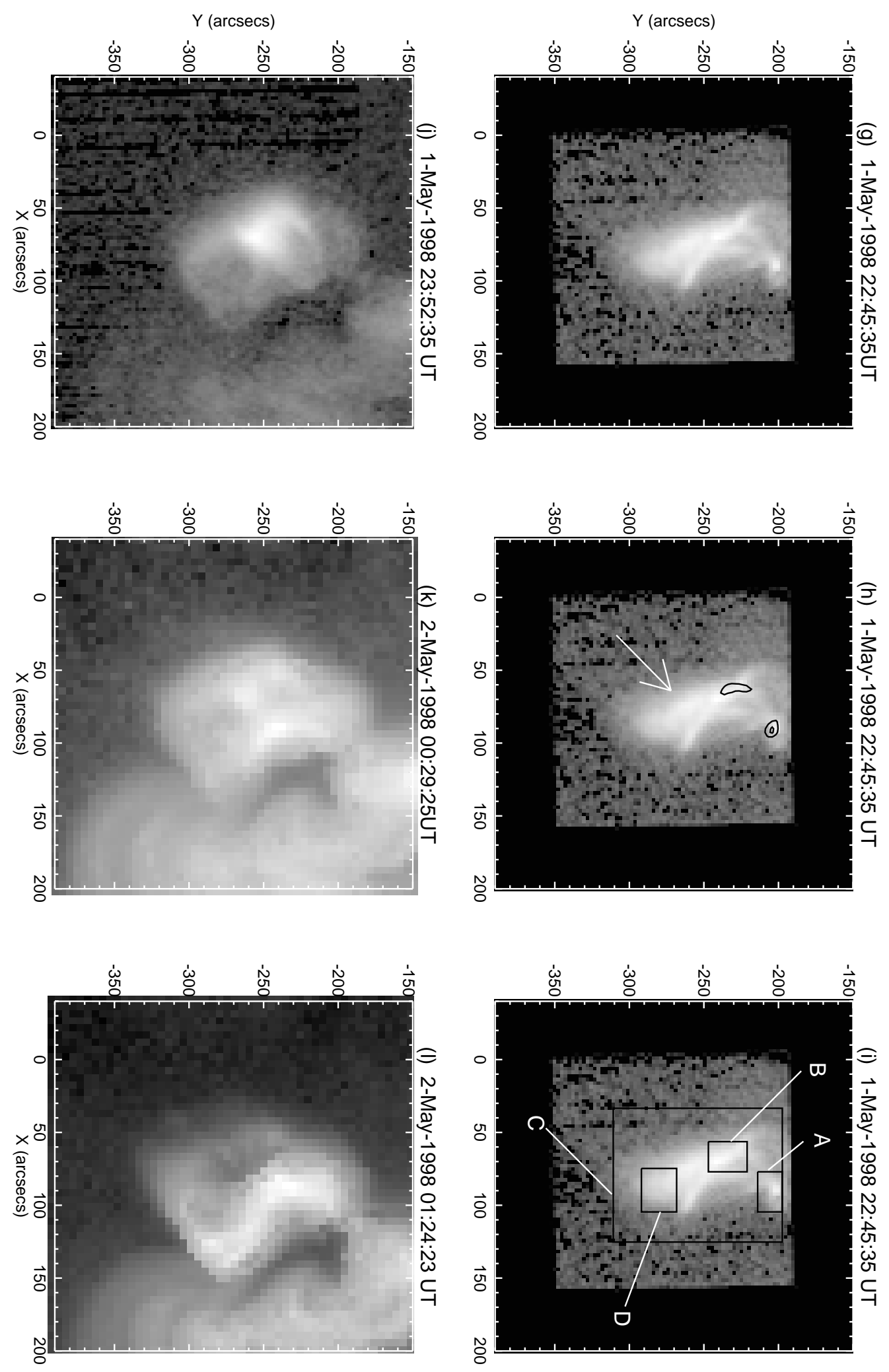

Fig. 6.- (Continued.) 

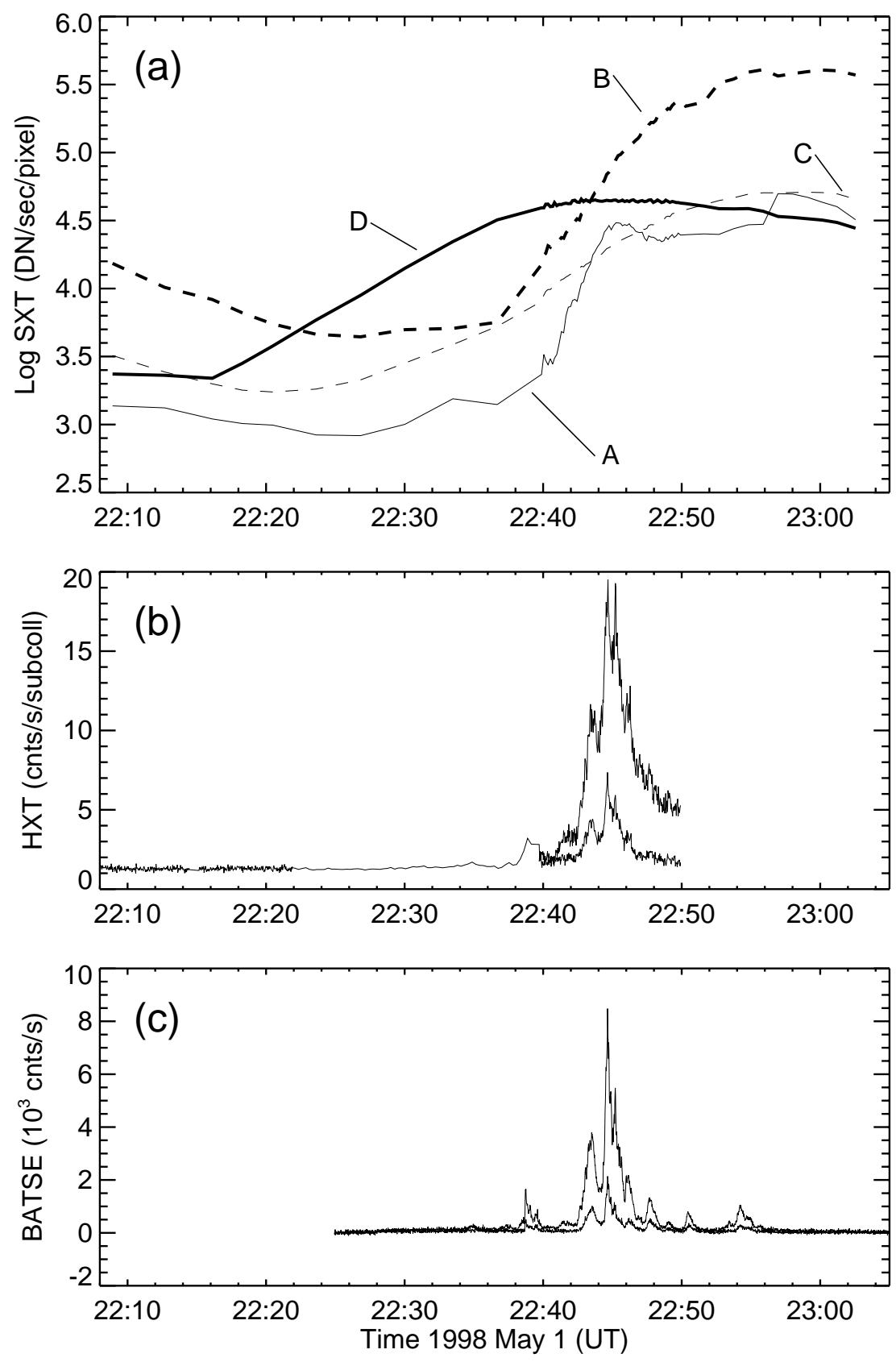

Fig. 7.- 

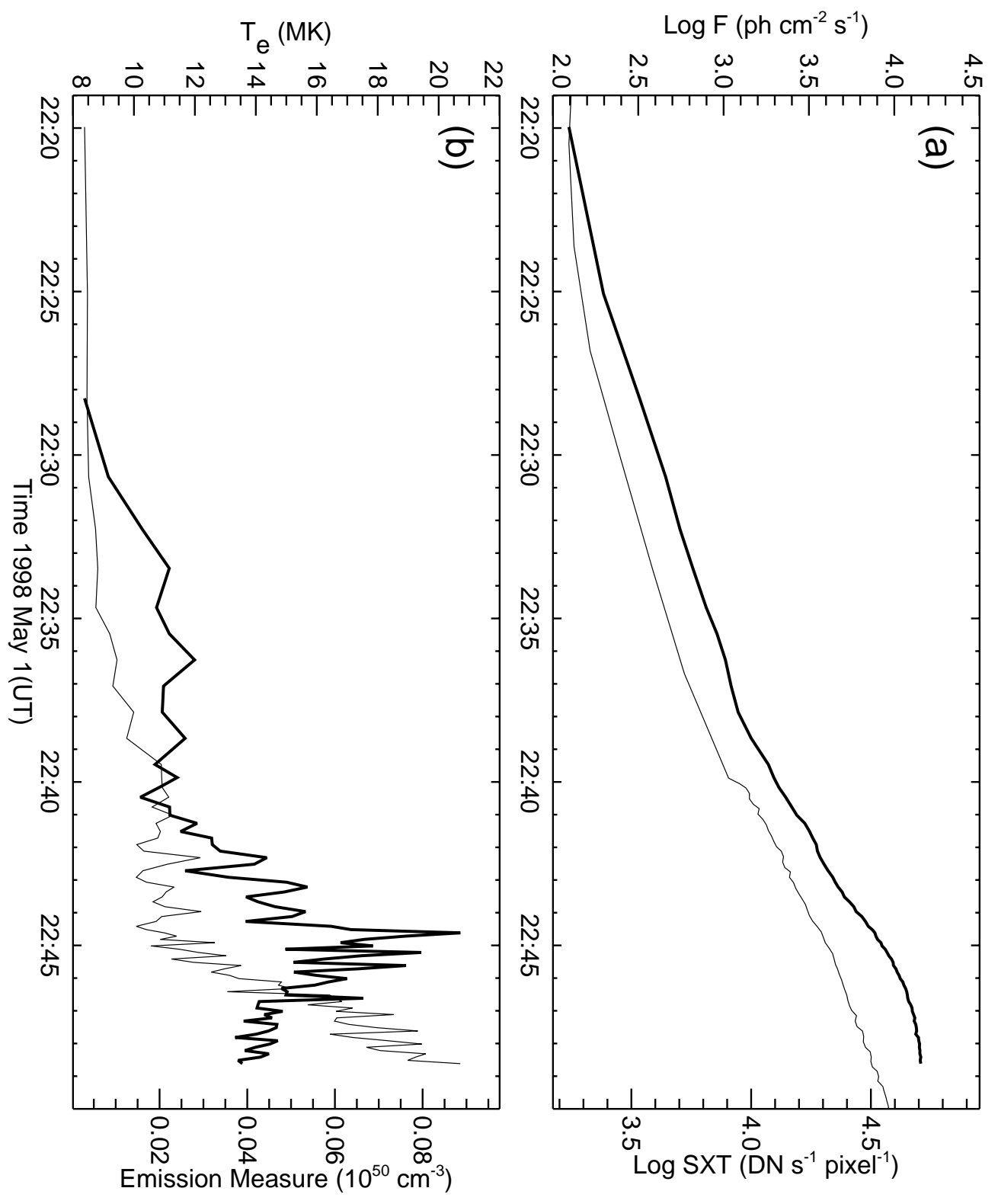

Fig. 8.- 

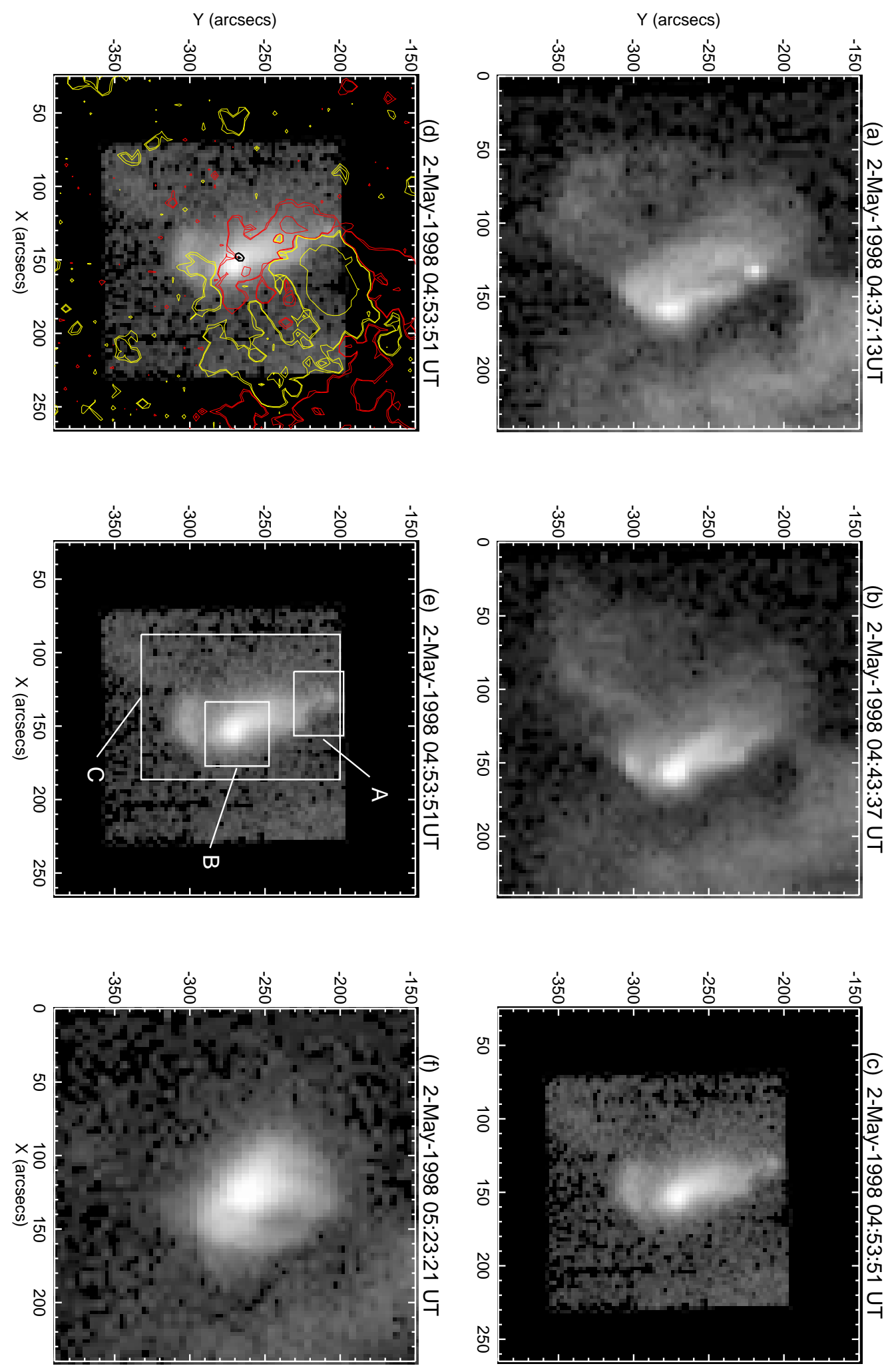

Fig. 9.- 

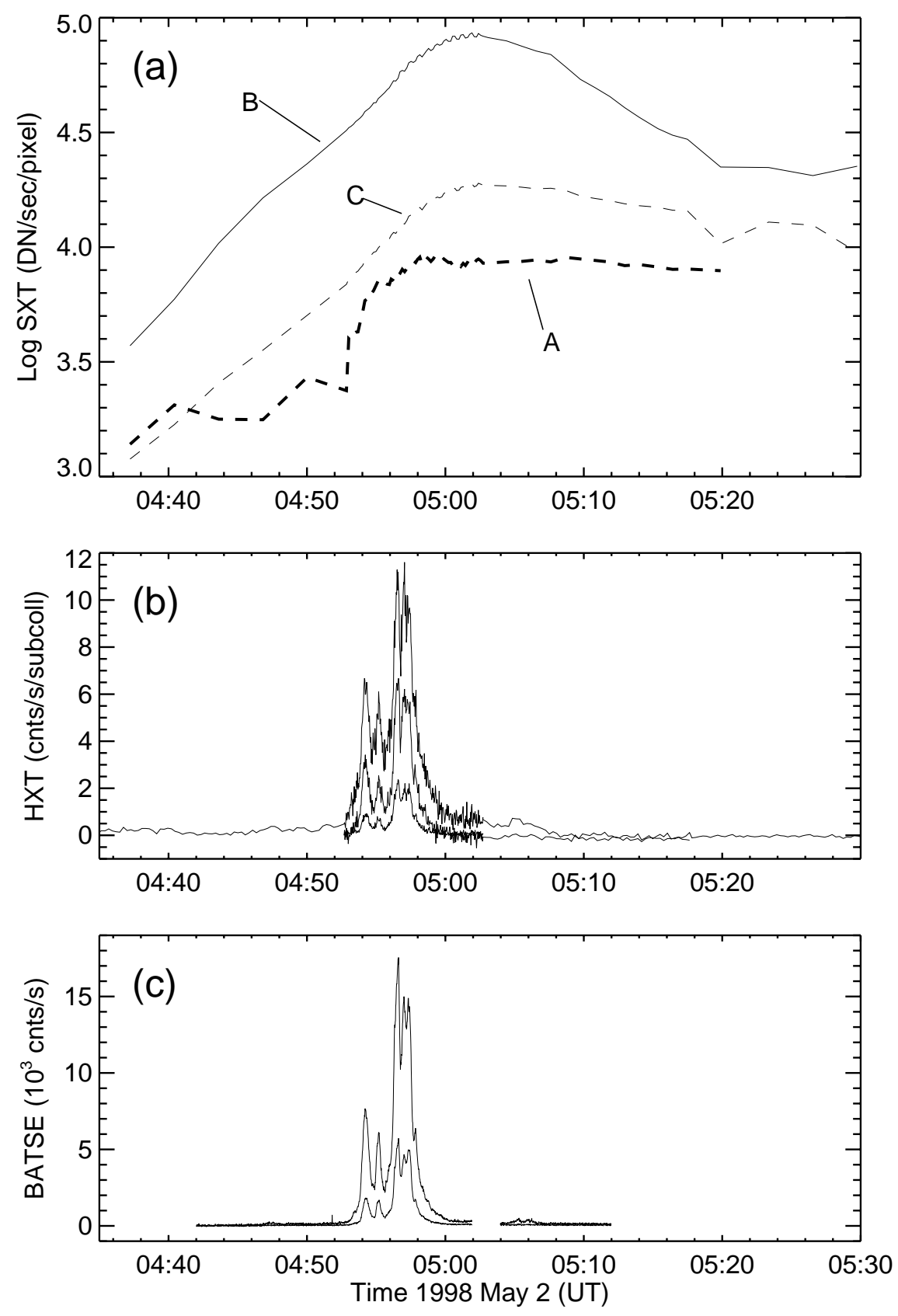

Fig. 10.- 


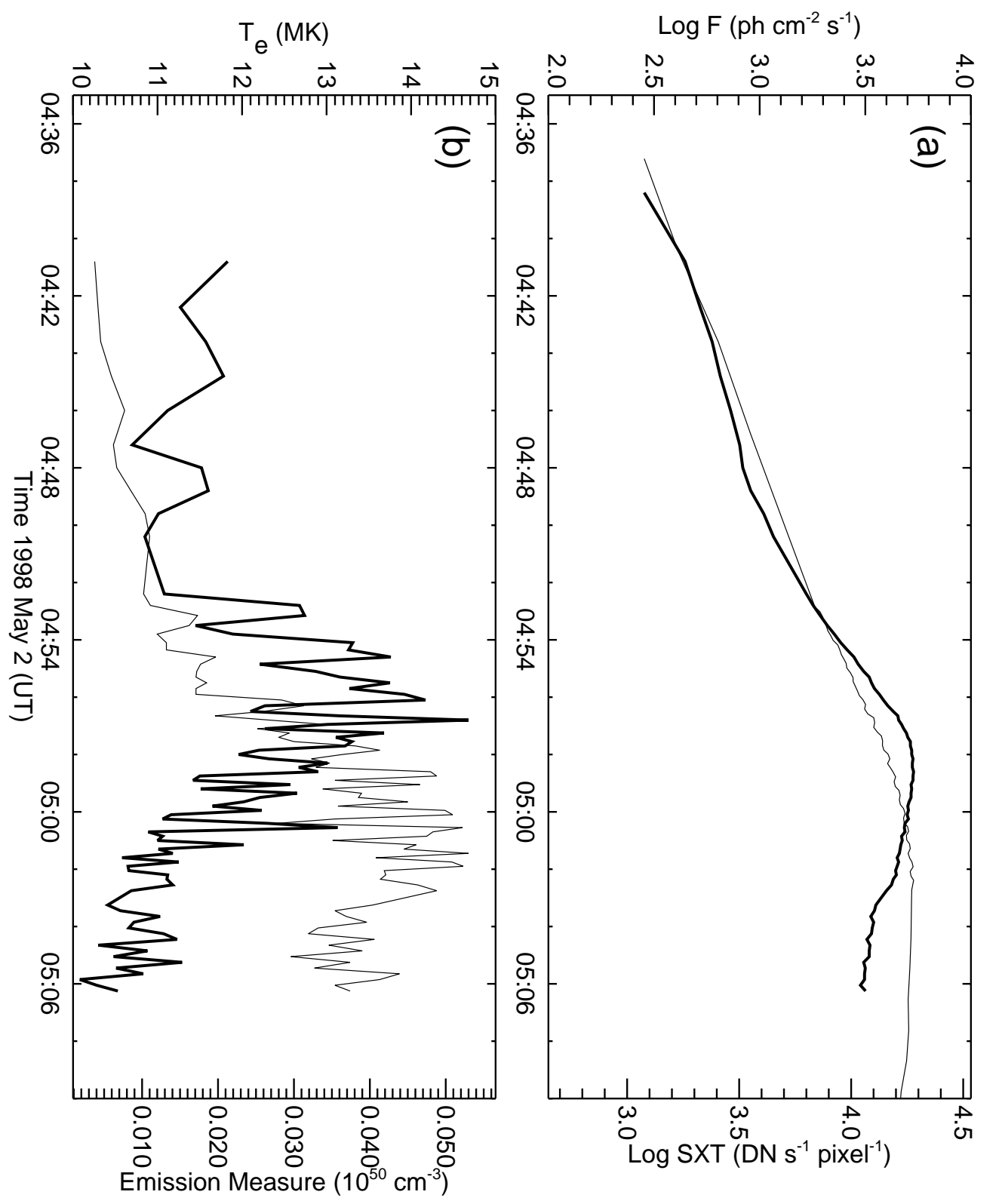

Fig. 11.- 